\title{
Research Paper: Immediate Effect of Respiratory Muscle Sprint-Interval Training (RMSIT) on the Plantar Pressure Variables in Athletes with Chronic Low Back Pain: A Randomized Controlled Trial
}

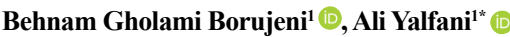

1. Department of Corrective Exercises and Sport Injury, Faculty of Physical Education and Sport Sciences, Bu-Ali Sina University, Hamadan, Iran.

\begin{tabular}{|c|c|}
\hline $\begin{array}{l}\text { Use evour device to scan } \\
\text { and read the article online }\end{array}$ & Chtation: Gholami Borujen B, Yalfani A. Immediate Effect of Respiratory Muscle Sprint-Interval Training (RMSIT) on the \\
\hline 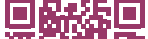 & Plantar Pressure Variables in Athletes With Chronic Low Back Pain: A Randomized Controlled Trial. Iranian Rehabilitation \\
\hline & Journal. 2019; 17(3):271-278. http://dx.doi.org/10.32598/irj.17.3.271 \\
\hline 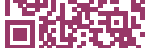 & dol ${ }^{\circ}$ ttp://dx.doi.org/10.32598/irj.17.3.271 \\
\hline
\end{tabular}

\section{(i) (3)}

Article info:

Received: 27 Dec 2019

Accepted: 25 May 2019

Available Online: 01 Sep 2019

Keywords:

Respiratory muscle, Sprintinterval training, Plantar pressure, Low back pain, Athlete

\section{ABSTRACT}

Objectives: The respiratory function is often overlooked in people with Low Back Pain (LBP), while it is one of the factors for the continuation and recurrence of lumbar spine injury. Aim of this study was survay the immediate effect of Respiratory Muscle Sprint-Interval Training (RMSIT) on the plantar pressure variables of athletes with LBP.

Methods: This randomized controlled trial was conducted on two groups with LBP, one experimental group (24 patients) and one control group (23 patients). The plantar pressure variables were recorded using a plantar pressure measurement device. The experimental group performed the RMSIT protocol. The RMSIT was fulfilled as six sets of 30-second deep breathing exercises (with two minutes of rest between sets) with constant tidal volume, maximum breathing rate, and added resistance. The paired t-test and one-way covariance tests were used to analyze the data

Results: The results of $(95 \%)$ confidence ellipse showed that RMSIT caused a significant decrease in the amount area of sway, but the exercise did not make a significant difference in the length of minor axis and length of the major axis. The results of the Center of Presure (COP) factors showed that the training program significantly reduced the amount of path length, the standard deviation in the $\mathrm{Y}$ axis, but the results did not show a significant difference in the velocity and standard deviation in the $\mathrm{X}$ axis in the experimental group. The results demonstrated no significant difference between the experimental and control group in the plantar pressure symmetry indices.

Discussion: It can be concluded that, these exercises can be used to reduce some postural oscillation indices in athletes with LBP.

* Corresponding Author:

Ali Yalfani, PhD.

Address: Department of Corrective Exercises and Sport Injury, Faculty of Physical Education and Sport Sciences, Bu-Ali Sina University, Hamadan, Iran Tel: +98 (813) 8381422

E-mail:ali_yalfani@yahoo.com 


\section{Highlights}

- Immediate respiratory training changed some the plantar pressure variables.

- Immediate respiratory training reduced the center of pressure sway.

- Respiratory training not affects on plantar pressure symmetry indices.

- Immediate respiratory training improves postural control.

\section{Plain Language Summary}

The people with Low back pain are suffering from impaired coordination between the trunk and the lower extremity, as well as balance defects. In this study, respiratory muscle training was used to reduce postural sway. The results of this study showed that respiratory training can reduce balance defects. Therefore this training method can be used for the rehabilitation of people with LBP and can be used for the balance improvement.

\section{Introduction}

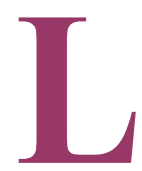

ow Back Pain (LBP) is one of the most common diseases caused by exercise $[1$, 2]. The people with LBP are suffering from impaired coordination between the trunk and the lower extremity [2]. as well as postural control changes [3]. Among the symptoms of chronic LBP disability, abnormal respiratory patterns and its various methods are of the most common symptoms that are often underestimated [4]. In general, movements of the chest and ribs are interconnected with spine[4]. Imbalance in the muscles that attached to spinal leads to spinal malalignment and these changes in most people cause pain in the cervical, thoracic and lumbar region [4]. The Chest Wall Expansion (CWE) is due to the cocontraction or bracing of erector spinae and abdominal muscles. The respiratory function is often overlooked in people with LBP, while it is one of the factors for the continuation and recurrence of lumbar spine injury [4].

Respiratory exercises have the advantage for posture because, facilitating the uplift and rotation of the ribs and this develops favorable conditions for the CWE and is related to the length of the muscles, hereby providing a mechanical advantage for postural alignment [5]. Activating respiratory muscles causes an increase in intra-abdominal pressure caused by transverse abdominis muscles contraction and thus reduces LBP [6].

Squat is an important component of strengthening training in athletes. In addition, the squat as a movement pattern is necessary in many movements, such as sitting, lifting and many exercises. The National Academy of
Sports Medicine (NASM) has proposed overhead squat as a useful indicator for the quality of movement [7].

Given the theoretical foundations, it can be concluded that probably activating respiratory muscles through effecting local stabilizers, especially Transverse Abdominis (TVA) and diaphragm as important respiratory muscles, and changing posture have different effects on plantar pressure and postural control. Investigations in this problem are limited, and no study was found on the effect of these exercises on plantar pressure. Therefore, the present study was conducted to evaluate the immediate effect of Respiratory Muscle Sprint-Interval Training (RMSIT) on plantar pressure variables of athletes with chronic LBP when implementing overhead squat. The study examined the impact of immediate respiratory training to be able to introduce a protocol that reduces the likelihood of injury in each training session or competition.

\section{Methods}

This randomized controlled trial was conducted on two groups, one experimental group and one control group. The statistical population of this study was strength athletes in Hamadan, Iran, with an age range of 18 to 25 years old. This study was conducted in March 2018. Those who had more than three months of LBP with an undetectable etiology were selected as the subjects with chronic nonspecific LBP [8]. According to the G*power software, 47 people were selected as the statistical sample available in accordance with the inclusion criteria [9].Of these 47, randomly simple, 24 patients were selected as experimental group and 23 patients as control group (Figure 1). 


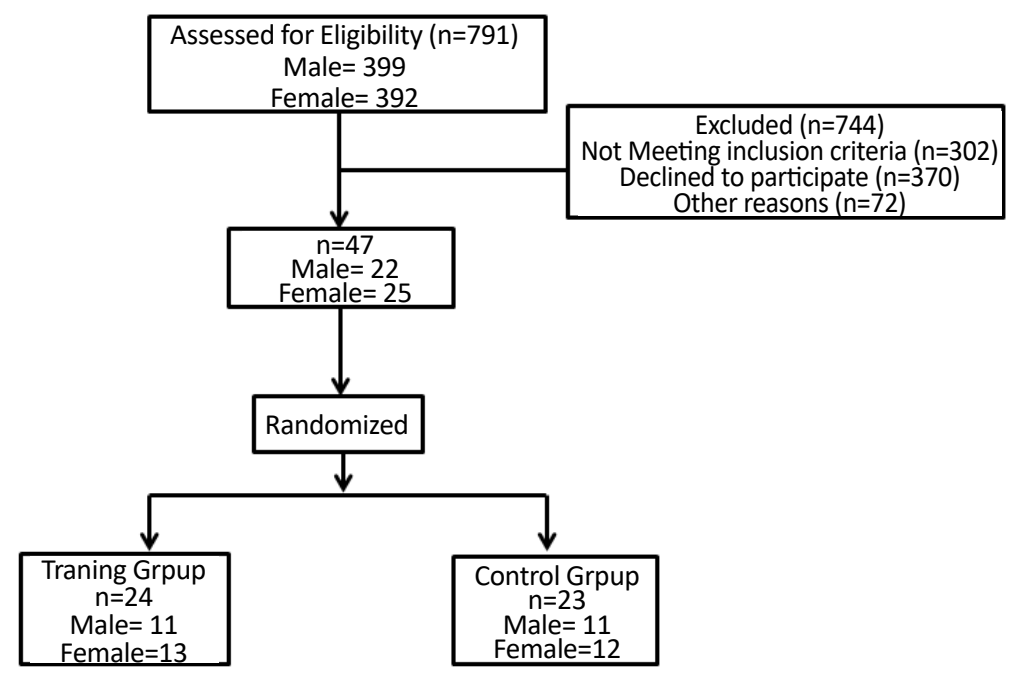

Figure 1. Sample selection diagram

Iranian Rehabilitation Journal

It should be noted that the subjects were selected after taking the necessary examinations to participate in this study. The athletes in this study were selected from individuals who had at least three years of continuous and regular attendance in resistance and weight training exercises, and had at least three training sessions each week with duration of each session more than an hour. Participants had no history of surgery, fracture, burn, neuromuscular problems and injury in spine and lower extremity, and had no malalignments in the lumbar or pelvic region and the lower limbs.

The plantar pressure variables were recorded using a plantar pressure measurement device (model Foot pressing FDM-S, Zebris Co. Germany) [10]. For respiratory muscles strengthening was used of Power breathe Spirometer (Figure 2).

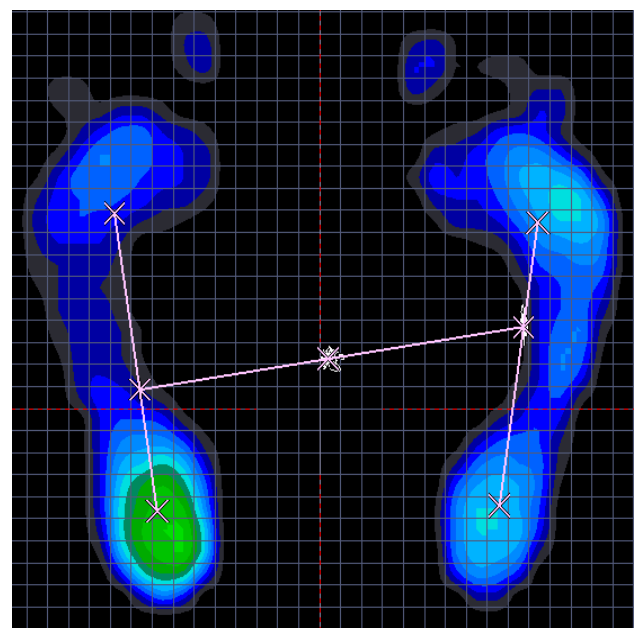

Figure 2. Average force distribution and COP Sway
Before the test, the participants performed warming up for 6 minutes, including 3 minutes of warming up with an ergometer at a constant speed and resistance, and 3 minutes of full-body stretching movement.

To perform the test, the subjects stood as the feet shoulder width apart and straight to the front. The ankle and foot were in a neutral position. The participant was asked to hold the hands up and extend the elbows completely, placing the arms symmetrically beside of head. The person was in the overhead squat position (the knee angle was 90 degrees), the feet were forward and the knees were aligned with the feet (second and third toes). The tibia and arms were aligned with the trunk. The heels were in contact with the ground, the feet were also at $10^{\circ}$ Foot Progression Angle (FPA) (the angle between the heel center and the second toe) [7]. The duration of the test was 30 seconds.

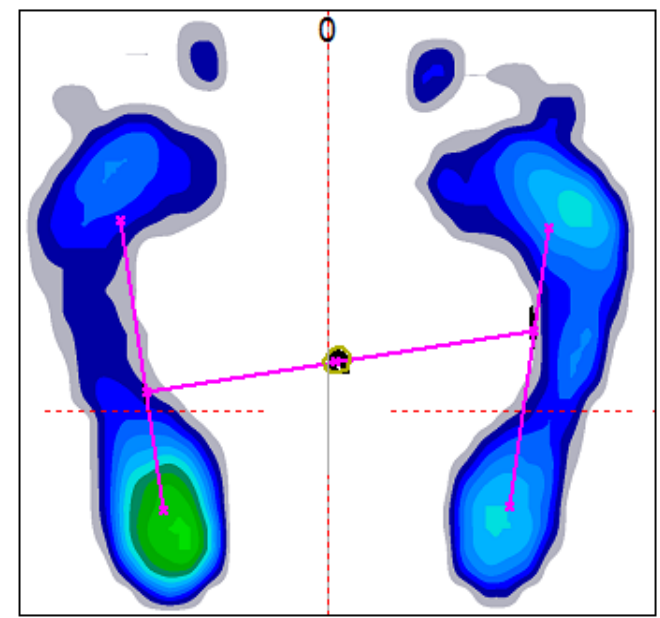

Iranian Rehabilitation Journal 


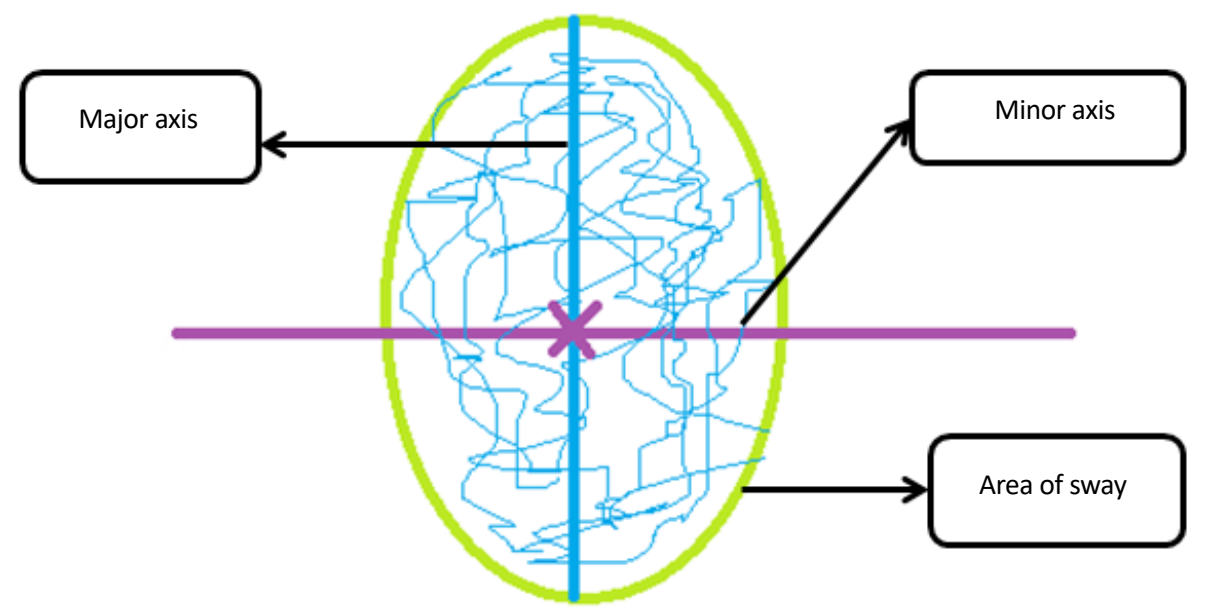

Figure 3. 95\% Confidence ellipse

Iranian Rehabilitation Journal

After recording the pre-test data, the participants of the experimental group performed the RMSIT protocol, followed by post-test after two minutes. The RMSIT was fulfilled as six sets of 30-second deep breathing exercise (with two minutes rest between sets) with constant tidal volume, maximum breathing rate and added resistance [11]. In the control group, they had no activity for 15 minutes after pre-test (similar to the protocol performed by the experimental group) and then the post-test was completed. The plantar pressure variables were analyzed using the Win FDM-S stance (version 01.02.09) software. The symmetry index between two feet was calculated by the equation of, and symmetry index between forward and backward of foot calculated by [10].

$$
\begin{aligned}
& S I=\frac{\text { right force }}{\text { right force }+ \text { left force }} \\
& S I=\frac{\text { forward force }}{\text { forward force }+ \text { backward force }}
\end{aligned}
$$

Shapiro-Wilk test was used to determine the normal distribution of data. Paired t-test and one-way covariance tests were used to analyze the effect of RMSIT on plantar pressure variables. Data were analyzed by SPSS V. 20 software and the significance level was $\alpha=0.05$.

\section{Results}

The Shapiro-Wilk test results in each group showed that the distribution of data in the research variables in that group was normal ( $p>0.05)$. Demographic information including: age, Height, Mass, BMI, Lumbar Arch Index and Pain index are listed in Table 1.

The paired t-test results (Table 2) showed that the RMSIT among the (95\%) confidence ellipse (Figure 3) parameters only caused a significant decrease in area of sway from the pre-test to post-test in the experimental group. The paired t-test results showed no significant differences in length of minor axis and length of major axis between pre-test and post-test in the experimental group. The paired t-test results between the pre-test and post-

Table 1. Demographic information

\begin{tabular}{ccccccccc}
\hline \multirow{2}{*}{ Group } & Gender & $\mathbf{n}$ & \multicolumn{7}{c}{ Mean \pm SD } \\
\cline { 5 - 9 } & & & Age, $\mathbf{y}$ & Height $(\mathbf{c m})$ & Mass (kg) & BMI & LAl (deg) & Pain Index \\
\hline \multirow{2}{*}{ Training } & Male & 11 & $20.14 \pm 1.34$ & $175.57 \pm 9.84$ & $70.01 \pm 10.66$ & $22.57 \pm 2.30$ & $31.02 \pm 5.12$ & $4.84 \pm 1.22$ \\
& Female & 13 & $22.44 \pm 2.18$ & $162.59 \pm 6.78$ & $57.95 \pm 9.29$ & $21.86 \pm 3.37$ & $31.22 \pm 4.76$ & $5.42 \pm 1.30$ \\
& Male & 11 & $22.50 \pm 1.29$ & $175.25 \pm 3.30$ & $67.12 \pm 6.17$ & $22.12 \pm 2.65$ & $28.01 \pm 4.54$ & $4.70 \pm 1.28$ \\
\multirow{2}{*}{ Control } & Female & 12 & $22.20 \pm 1.64$ & $160.36 \pm 4.97$ & $54.54 \pm 7.83$ & $21.18 \pm 2.82$ & $30.20 \pm 6.34$ & $4.88 \pm 1.22$ \\
\hline
\end{tabular}

LAI: Lumbar Arch Index

Iranian Rehabilitation Journal 
Table 2. Paired t-test and One-Way Covariance Analysis in results of (95\%) confidence ellipse

\begin{tabular}{|c|c|c|c|c|c|c|c|}
\hline \multirow{2}{*}{ Variable } & \multirow{2}{*}{ Group } & \multicolumn{2}{|c|}{ Mean $\pm S D$} & \multirow{2}{*}{ Sig (t-Paired) } & \multirow{2}{*}{$\mathbf{F}$} & \multirow{2}{*}{ Sig } & \multirow{2}{*}{$\begin{array}{c}\text { Partial Eta } \\
\text { Squared }\end{array}$} \\
\hline & & BT & AT & & & & \\
\hline \multirow{2}{*}{$\begin{array}{l}\text { Length of minor } \\
\quad \text { axis }(\mathrm{mm})\end{array}$} & Training & $22.33 \pm 15.32$ & $16.25 \pm 12.25$ & 0.153 & \multirow{2}{*}{2.16} & \multirow{2}{*}{0.155} & \multirow{2}{*}{0.090} \\
\hline & Control & $19.11 \pm 12.17$ & $23.20 \pm 14.91$ & 0.321 & & & \\
\hline \multirow{2}{*}{$\begin{array}{l}\text { Length of major } \\
\text { axis }(\mathrm{mm})\end{array}$} & Training & $51.38 \pm 24.07$ & $45.68 \pm 29.45$ & 0.702 & \multirow{2}{*}{0.00} & \multirow{2}{*}{0.990} & \multirow{2}{*}{0.00} \\
\hline & Control & $46.28 \pm 27.01$ & $49.94 \pm 30.37$ & 0.467 & & & \\
\hline \multirow{3}{*}{ Area $\left(\mathrm{mm}^{\wedge} 2\right)$} & Training & $928.73 \pm 240.06$ & $303.63 \pm 129.35$ & $0.011^{*}$ & \multirow{3}{*}{7.03} & \multirow{3}{*}{$0.015^{*}$} & \multirow{3}{*}{0.242} \\
\hline & & & & & & & \\
\hline & Control & $839.12 \pm 207.59$ & $967.83 \pm 288.08$ & 0.270 & & & \\
\hline
\end{tabular}

*Indicates significant difference $(\mathrm{P}<0.05)$; BT: Before Training; AT: After Training

Iranian Rehabilitation Journal

test scores in the $(95 \%)$ confidence ellipse parameters in the control group found no significant difference. The one-way covariance test results revealed a significant difference in the area of way index between the control and the experimental group. However, the one-way covariance test results exhibited no significant difference in the length of major axis and length of minor axis between the control and experimental group.

The paired t-test results (Table 3) reported that the RMSIT significantly reduced some of the center of pressure oscillation factors including path length and standard deviation in $\mathrm{Y}$ axis in the experimental group from pretest to post-test, but the paired t-test results underlined no significant difference in the velocity and standard deviation in X axis between pre-test and post-test in the experimental group. The paired t-test showed no significant difference in the scores of the center of pressure oscillation factors between pre-test and post-test in the control group. Moreover, one-way covariance test results showed significant differences in path length and standard deviation in $\mathrm{Y}$ axis between control group and experimental group, but one-way covariance test results found no significant difference in scores of velocity and standard deviation in $\mathrm{X}$ axis between the control and the experimental groups.

According to the paired t-test results (Table 4) in the experimental and control groups, there was no significant difference in the plantar pressure symmetry between pre-test and post-test. Based on one-way covariance test results, there was no significant difference in plantar pressure symmetry between experimental and the control groups.

\section{Discussion}

The results of this study showed that respiratory muscle training changed some the plantar pressure variables in athletes with LBP. The present study is consistent with some of the previous studies in terms of the effect of respiratory training and activation of these muscles, which are mainly local muscles of core region $[12,13]$. The postural control and respiration are mechanically and neuromuscularly dependent on each other, both

Table 3. Paired t-test and one-way covariance analysis in results of (COP) sway

\begin{tabular}{|c|c|c|c|c|c|c|c|}
\hline \multirow{2}{*}{ Variable } & \multirow{2}{*}{ GROUP } & \multicolumn{2}{|c|}{ Mean $\pm S D$} & \multirow{2}{*}{ Sig (t-Paired) } & \multirow{2}{*}{$\mathbf{F}$} & \multirow{2}{*}{ Sig } & \multirow{2}{*}{$\begin{array}{c}\text { Partial Eta } \\
\text { Squared }\end{array}$} \\
\hline & & BT & AT & & & & \\
\hline \multirow{2}{*}{ Path Length (mm) } & Training & $906.45 \pm 253.48$ & $484.08 \pm 218.78$ & $0.011^{*}$ & \multirow{2}{*}{10.84} & \multirow{2}{*}{$0.003^{*}$} & \multirow{2}{*}{0.330} \\
\hline & Control & $767.81 \pm 252.89$ & $795.45 \pm 110.34$ & 0.301 & & & \\
\hline \multirow{2}{*}{ Velocity (mm/ sec) } & Training & $34.20 \pm 17.61$ & $33.35 \pm 18.18$ & 0.929 & \multirow{2}{*}{0.75} & \multirow{2}{*}{0.396} & \multirow{2}{*}{0.033} \\
\hline & Control & $32.90 \pm 11.14$ & $42.30 \pm 19.80$ & 0.252 & & & \\
\hline \multirow{2}{*}{ Standard Diviation X $(\mathrm{mm})$} & Training & $55.18 \pm 26.06$ & $37.10 \pm 23.99$ & 0.719 & \multirow{2}{*}{0.29} & \multirow{2}{*}{0.596} & \multirow{2}{*}{0.013} \\
\hline & Control & $45.48 \pm 15.21$ & $52.87 \pm 18.65$ & 0.109 & & & \\
\hline \multirow{2}{*}{ Standard Diviation $\mathrm{Y}(\mathrm{mm})$} & Training & $55.18 \pm 26.06$ & $37.10 \pm 23.99$ & $0.013^{*}$ & \multirow{2}{*}{4.71} & \multirow{2}{*}{$0.041^{*}$} & \multirow{2}{*}{0.176} \\
\hline & Control & $51.95 \pm 26.40$ & $56.17 \pm 28.31$ & 0.263 & & & \\
\hline
\end{tabular}

*Indicates significant difference $(\mathrm{P}<0.05)$; BT: Before Training; AT: After Training

Iranian Rehabilitation Journal 
Table 4. Paired t-test and one-way covariance analysis in results of symmetry of plantar pressure

\begin{tabular}{|c|c|c|c|c|c|c|c|}
\hline \multirow{2}{*}{ Variable } & \multirow{2}{*}{ GROUP } & \multicolumn{2}{|c|}{ Mean \pm SD } & \multirow{2}{*}{ Sig (t-Paired) } & \multirow{2}{*}{$\mathbf{F}$} & \multirow{2}{*}{ Sig(F) } & \multirow{2}{*}{$\begin{array}{l}\text { Partial Eta } \\
\text { Squared }\end{array}$} \\
\hline & & BT & AT & & & & \\
\hline \multirow{2}{*}{ S of Left \& Right } & Training & $0.487 \pm 0.102$ & $0.481 \pm 0.07$ & 0.833 & \multirow{2}{*}{3.22} & \multirow{2}{*}{0.086} & \multirow{2}{*}{0.128} \\
\hline & Control & $0.519 \pm 0.09$ & $0.569 \pm 0.10$ & 0.214 & & & \\
\hline \multirow{2}{*}{$S$ of $F \& B$ (Right) } & Training & $0.570 \pm 0.28$ & $0.457 \pm 0.264$ & 0.145 & \multirow{2}{*}{0.86} & \multirow{2}{*}{0.363} & \multirow{2}{*}{0.038} \\
\hline & Control & $0.478 \pm 0.29$ & $0.485 \pm 0.302$ & 0.668 & & & \\
\hline \multirow{2}{*}{ S of $F \& B$ (Left) } & Training & $0.668 \pm 0.28$ & $0.614 \pm 0.22$ & 0.194 & \multirow{2}{*}{0.87} & \multirow{2}{*}{0.36} & \multirow{2}{*}{0.038} \\
\hline & Control & $0.499 \pm 0.29$ & $0.573 \pm 0.26$ & 0.372 & & & \\
\hline
\end{tabular}

Iranian Rehabilitation Journal

*Indicates significant difference (P<0.05); BT: Before Training; AT: After Training; S: Symmetry; F\&B. Fore \& Back of foot

systems involve diaphragm, TVA, intercostal muscles, internal oblique muscles and pelvic floor muscle [14]. These muscles play a special role in voluntary breathing, which means that the TVA along with multifidus is active in stabilizing the lumbar region $[12,15]$.

As well as Vostatek, et al. (2013) stated that the diaphragm has both respiratory and postural functions [16]. Focus of the respiratory training in the present study was the deep and local muscles of the core region. Hodges and Gandevia (2000) also argued that inspiratory muscles, especially the diaphragm, play a key role in controlling the spine, which are vital during postural control [14]. Janssens et al. (2015) also found that inspiratory muscle training improved postural control in people with LBP and decreased LBP severity significantly [13].

Our results demonstrated no significant difference between the experimental and control group in the plantar pressure symmetry indices. No found study that survey the effect of respiratory training on plantar pressure variables. But according to the results of studies, entire movement system covers many body parts, and that a proper alignment to the line of gravity ensures good posture; any irregularity in this alignment can cause changes in the adjacent segments and other components or even changes in the activity of body systems and organs $[17,18]$. Studies have shown that deep muscles activity improves body alignment, but the exercises used in the above studies were long-term and effective on spinal alignment [19].

Also, the alteration in the alignment of the body affects the plantar pressure [20]. Anukoolkarn et al. (2015) stated that the pressures on the plantar surface were unequally distributed in subjects with chronic non-specific low back pain [21]. In the present study, the RMSIT had no effect on right-left plantar pressure symmetry and also fore and back plantar pressure symmetry, probably due to short duration of exercise that did not have an impact on body alignment. It is likely that long-term training of these muscles will improve plantar pressure symmetry by improving posture.

One of the limitations of the research was that everyone had $10^{\circ}$ Foot Progression Angle (FPA), whereas people may put feet at different angles while training at the gyms. Therefore, the same FPA and squat angle were considered for all athletes in the present study to homogenize the conditions in all subjects in both pre-test and post-test. Another limitation of this study was to investigate the immediate effect of this training, whereas longterm exercises may provide better effects. Therefore, it is suggested to evaluate the long-term effects of these exercises in order to accurately introduce these exercises in the postural control and improving the plantar pressure symmetry. To introduce a protocol that reduces the likelihood of injury in each training session or competition this study survay the effect of immediate training instead of a long-term training protocol.

\section{Conclusion}

RMSIT protocol in the athletes with chronic LBP could have positive effects on the some of the center of pressure oscillation factors, but had no impacts on other center of pressure oscillation factors and plantar pressure symmetry parameters. As a result, these exercises can be used to reduce some postural oscillation indices in athletes. However, given the insignificant effect of these exercises on many study factors, as well as limited report in this problem, further studies are needed to examine the effect of these exercises (as both long-term and immediate) on plantar pressure variables. 


\section{Ethical Considerations}

\section{Compliance with ethical guidelines}

Before entering the lab, the subjects studied the written consent form and then signed it. This study was approved and registered at the Ethics Committee of Hamadan University of Medical Sciences (Code: IR.UMSHA. REC.1396.844). The data extract center was Bu-Ali Sina University Sport Rehabilitation Lab.

\section{Funding}

This research is extracted from $\mathrm{PhD}$ dissertation of First Author, Departemant of Corrective Exercises \& Sport Injury, Faculty of Physical Education and Sport Sciences at Bu-Ali Sina University, Hamadan. This research did not receive any specific grant from funding agencies in the public, commercial, or not-forprofit sectors.

\section{Authors' contributions}

Reading and approve the final version of the manuscript, agreeance with the order of presentation of the authors: Behnam Gholami Borujeni, Ali Yalfani.

\section{Conflict of interest}

The authors declared no conflict of interest.

\section{References}

[1] Dehkordi F, Khankeh HR, Hassani Mehraban A, Hosseini SA. The impact of chronic low back pain on daily occupations: A qualitative study in Iranian context. Iranian Rehabilitation Journal. 2016; 14(1):15-22. [DOI:10.15412/J.IRJ.08140103]

[2] Herndon CL, Horodyski M, and Vincent HK. Acute effects of anesthetic lumbar spine injections on temporal spatial parameters of gait in individuals with chronic low back pain: A pilot study. Gait \& Posture. 2017; 58(1):369-73. [DOI:10.1016/j. gaitpost.2017.08.016] [PMID]

[3] Schelldorfer S, Ernst MJ, Rast FM, Bauer CM, Meichtry A, Kool J. Low back pain and postural control, effects of task difficulty on centre of pressure and spinal kinematics. Gait \& Posture. 2015; 41(1):112-8. [DOI:10.1016/j.gaitpost.2014.09.004] [PMID]

[4] Babina R, Mohanty PP, Pattnaik M. Effect of thoracic mobilization on respiratory parameters in chronic non-specific low back pain: A randomized controlled trial. Journal of Back and Musculoskeletal Rehabilitation. 2016; 29(3):587-95. [DOI:10.3233/BMR-160679] [PMID]

[5] Lee M, Lau H, Lau T. Sagittal plane rotation of the pelvis during lumbar posteroanterior loading. Journal of Manipu- lative and Physiological Therapeutics. 1994; 17(3):149-55. [PMID]

[6] Hodges PW, Gandevia S. Activation of the human diaphragm during a repetitive postural task. The Journal of Physiology. 2000; 522(1):165-75. [DOI:10.1111/j.1469-7793.2000. t01-1-00165.xm] [PMID] [PMCID]

[7] Bishop C, Edwards M, Turner A. Screening movement dysfunctions using the overhead squat. The Journal of Strength and Conditioning Research. 2016; 42:22-30.

[8] Last AR, Hulbert K. Chronic low back pain: Evaluation and management. American Family Physician. 2009; 79(12):106774. [DOI:10.1080/20786204.2010.10873969]

[9] Faul F, Erdfelder E, Lang A-G, Buchner A. G* Power 3: A flexible statistical power analysis program for the social, behavioral, and biomedical sciences. Behavior Research Methods. 2007; 39(2):175-91. [DOI:10.3758/BF03193146] [PMID]

[10] Braun BJ, Veith NT, Hell R, Döbele S, Roland M, Rollmann $\mathrm{M}$, et al. Validation and reliability testing of a new, fully integrated gait analysis insole. Journal of Foot and Ankle Research. 2015; 8(54):1-7. [DOI:10.1186/s13047-015-0111-8] [PMID] [PMCID]

[11] Wüthrich TU, Marty J, Benaglia P, Eichenberger PA, Spengler CM. Acute effects of a respiratory sprint-interval session on muscle contractility. Medicine and Science in Sports and Exercise. 2015; 47(9):1979-87. [DOI:10.1249/ MSS.0000000000000627] [PMID]

[12] Szczygieł E, Blaut J, Zielonka-Pycka K, Tomaszewski K, Golec J, Czechowska D, et al. The impact of deep muscle training on the quality of posture and breathing. Journal of Motor Behavior. 2018; 50(2):219-27. [DOI:10.1080/00222895.2 017.1327413] [PMID]

[13] Janssens, L, McConnell AK, Pijnenburg M, Claeys K, Goossens N, Lysens R, et al. Inspiratory muscle training af fects proprioceptive use and low back pain. Medicine \& Science in Sports \& Exercise. 2015; 47(1):12-9. [DOI:10.1249/ MSS.0000000000000385] [PMID]

[14] Hodges PW, Gandevia SC. Changes in intra-abdominal pressure during postural and respiratory activation of the human diaphragm. Journal of Applied Physiology. 2000; 89(3):967-76. [DOI:10.1152/jappl.2000.89.3.967] [PMID]

[15] Hodges P, Sapsford R, Pengel L. Postural and respiratory functions of the pelvic floor muscles. Neurourology and Urodynamics. 2007; 26(3):362-71. [DOI:10.1002/nau.20232] [PMID]

[16] Vostatek P, Novák D, Rychnovský T, Rychnovská S. Diaphragm postural function analysis using magnetic resonance imaging. PlOS One. 2013; 8(3):e56724. [DOI:10.1371/journal. pone.0056724] [PMID] [PMCID]

[17] Hadała M, Gryckiewicz S. Movement pattern and muscle balance as a source of lumbar spine health according to the concept of kinetic control. Polish Annals of Medicine. 2014; 21(2):152-7. [DOI:10.1016/j.poamed.2014.06.001]

[18] Claeys K, Dankaerts W, Janssens L, Pijnenburg M, Goossens N, Brumagne S. Young individuals with a more anklesteered proprioceptive control strategy may develop mild non-specific low back pain. Journal of Electromyography and Kinesiology. 2015; 25(2):329-38. [DOI:10.1016/j.jelekin.2014.10.013] [PMID] 
[19] Tsao H, Hodges PW. Persistence of improvements in postural strategies following motor control training in people with recurrent low back pain. Journal of Electromyography and Kinesiology. 2008; 18(4):559-67. [DOI:10.1016/j.jelekin.2006.10.012] [PMID]

[20] Anbarian M, Esmaeili H. Effects of running-induced fatigue on plantar pressure distribution in novice runners with different foot types. Gait \& Posture. 2016; 48:52-6. [DOI:10.1016/j. gaitpost.2016.04.029] [PMID]

[21] Anukoolkarn K, Vongsirinavarat M, Bovonsunthonchai S, Vachalathiti R. Plantar pressure distribution pattern during mid-stance phase of the gait in patients with chronic nonspecific low back pain. Journal of the Medical Association of Thailand. 2015; 98(9):896-901. [PMID] 